\title{
Physics-Based Passivity-Preserving Parameterized Model Order Reduction for PEEC Circuit Analysis
}

\author{
Francesco Ferranti, Member, IEEE, Giulio Antonini, Senior Member, IEEE, Tom Dhaene, Senior Member, IEEE, \\ Luc Knockaert, Senior Member, IEEE, and Albert E. Ruehli, Life Fellow, IEEE
}

\begin{abstract}
The decrease of IC feature size and the increase of operating frequencies require 3D electromagnetic methods, such as the Partial Element Equivalent Circuit (PEEC) method, for the analysis and design of high-speed circuits. Very large systems of equations are often produced by $3 \mathrm{D}$ electromagnetic methods and model order reduction (MOR) methods were proven to be very effective in combating such high complexity. During the circuit synthesis of large-scale digital or analog applications, it is important to predict the response of the circuit under study as a function of design parameters, such as geometrical and substrate features. Traditional MOR techniques perform model order reduction only with respect to frequency, therefore the computation of a new electromagnetic model and corresponding reduced model is needed each time a design parameter is modified, reducing the CPU efficiency. Parameterized model order reduction (PMOR) methods become necessary to reduce large systems of equations with respect to frequency and other design parameters of the circuit, such as geometrical layout or substrate characteristics.

We propose a novel PMOR technique applicable to PEEC analysis which is based on a parameterization process of matrices generated by the PEEC method and the projection subspace generated by a passivity-preserving MOR method. The proposed PMOR technique guarantees overall stability and passivity of parameterized reduced order models over a user defined range of design parameter values. Pertinent numerical examples validate the proposed PMOR approach.
\end{abstract}

Index Terms-Partial Element Equivalent Circuit method (PEEC), parameterized model order reduction (PMOR), interpolation, passivity.

\section{INTRODUCTION}

Electromagnetic (EM) methods [1]-[3] have become increasingly indispensable analysis and design tools for a variety of complex high-speed systems. The use of these methods usually results in very large systems of equations which are prohibitively expensive to solve. Hence, model order reduction (MOR) techniques are crucial to reduce the complexity of EM models and the computational cost of the simulations, while

Manuscript received March 2010.

Francesco Ferranti, Tom Dhaene and Luc Knockaert are with the Department of Information Technology (INTEC), at Ghent University - IBBT, Sint Pietersnieuwstraat 41, 9000 Ghent, Belgium, email: \{francesco.ferranti, tom.dhaene, luc.knockaert\}@ugent.be.

Giulio Antonini is with the UAq EMC Laboratory, Dipartimento di Ingegneria Elettrica e dell'Informazione, Università degli Studi dell'Aquila, Via G. Gronchi 18,67100, L’Aquila, Italy, e-mail: giulio.antonini@univaq.it.

Albert E. Ruehli is an Emeritus of the IBM T. J. Watson Research Center, Yorktown Heights, NY 10598, USA and an Adjunct Professor at the Missouri University of S\&T in Rolla, e-mail: albert.ruehli@gmail.com.

This work was supported by the Research Foundation Flanders (FWO) and by the Italian Ministry of University (MIUR) under a Program for the Development of Research of National Interest, (PRIN grant n. 2006095890). retaining the important physical features of the original system [4]-[7]. The development of a reduced order model (ROM) of EM systems has become a topic of intense research over the last years, with applications to vias, high-speed packages, interconnects, and on-chip passive components [8]-[11]. An increasing popularity among electromagnetic compatibility engineers has been achieved by the Partial Element Equivalent Circuit (PEEC) method, since it is able to transform the EM system under examination into a passive RLC equivalent circuit. PEEC uses a circuit interpretation of the Electric Field Integral Equation (EFIE) [12], thus allowing to handle complex problems involving EM fields and circuits [2], [13], [14]. Nonlinear circuit devices such as drivers and receivers are usually connected with PEEC equivalent circuits using a time domain circuit simulator (e.g. SPICE [15]). However, inclusion of the PEEC model directly into a circuit simulator may be computationally intractable for complex structures, because the number of circuit elements can be in the tens of thousands. In this case, a first solution consists in the use of fast multipole methods [16], [17]. The drawback of these techniques relies on the fact that they are dependent on the Green's function of the problem. Another option is represented by MOR techniques which are adopted to reduce the size of the PEEC model [7], [18], [19].

Traditional MOR techniques perform model reduction only with respect to frequency. However, during the circuit synthesis of large-scale digital or analog applications, it is also important to predict the response of the circuit under study as a function of design parameters, such as geometrical and substrate features. A typical design process includes optimization and design space exploration, and thus requires repeated simulations for different design parameter values. Such design activities call for parameterized model order reduction (PMOR) methods that can reduce large systems of equations with respect to frequency and other design parameters of the circuit, such as geometrical layout or substrate characteristics.

Over the years, a number of PMOR methods have been developed. In order to model and analyze interconnect behavior with process variations, various techniques have been proposed for variational interconnect order reduction [20], [21]. These approaches apply projection operator and generate reducedorder interconnect models. In addition, the projection subspace and/or the reduced-order system matrices are approximated as low-order polynomials of process parameters such that the process variation effects can be incorporated into the interconnect model. These process parameters, for example, can be the width and thickness of the interconnect metal wires. 
The authors in [22] propose to approximate the system transfer function by low-order polynomials of process parameters, instead of the projection subspace and/or the reduced order system matrices. The algorithm described in [22] computes the projection subspace and generates parameterized ROMs such that the multiparameter moments are matched. However, the structure of such method may present some computational problems, and the resulting parameterized ROMs usually suffer from oversize when the number of moments to match is high, either because high accuracy (order) is required or because the number of parameters is large. The Compact Order Reduction for parameterized Extraction (CORE) algorithm [23] applies a two-step explicit-and-implicit scheme for multiparameter moment matching. It is numerically stable, but unfortunately it does not preserve passivity. The Parameterized Interconnect Macromodeling via a two-directional Arnoldi process (PIMTAP) algorithm presented in [24] is numerically stable, preserves the passivity of parameterized RLC networks, but, such as all multiparameter moment-matching based PMOR techniques, it is suitable only to a low-dimensional design space.

This paper proposes a PMOR method applicable to PEEC analysis which is based on a parameterization process of matrices generated by the PEEC method and the projection subspace generated by a passivity-preserving MOR method. The Laguerre-SVD MOR method [19] is used in this paper. Overall stability and passivity of parameterized ROMs are guaranteed by construction over the design space of interest. PEEC models and parameterized ROMs describe an admittance $(\mathbf{Y})$ representation. However, it should be noted that the proposed PMOR technique is not bound to the Laguerre-SVD method, other passivity-preserving MOR techniques based on a projection subspace approach can be used, such as the PRIMA method [7].

The paper is organized as follows. Section II describes the modified nodal analysis (MNA) equations of the PEEC method. Section III describes the proposed PMOR method. Finally, some pertinent numerical examples validate the proposed technique in Section IV.

\section{PEEC FORMULATION}

The PEEC method [2] stems from the integral equation form of Maxwell's equations.

The main difference of the PEEC method with other integral equation based techniques such as the Method of Moments (MoM) [1] resides in the fact that it provides a circuit interpretation of the EFIE [12] in terms of partial elements, namely resistances, partial inductances and coefficients of potential. Thus, the resulting equivalent circuit can be studied by means of SPICE-like circuit solvers [15] in both time and frequency domain.

Over the years, several improvements of the PEEC method have been performed thus allowing to handle complex problems involving both circuits and electromagnetic fields [2], [13], [14], [25]-[28].

In the standard approach, volumes and surfaces are discretized into elementary regions, hexahedra and patches respectively [27] over which the current and charge densities are expanded into a series of basis functions. Pulse basis functions are usually adopted as expansion and weight functions. Such choice of pulse basis functions corresponds to assume constant current density and charge density over the elementary volume (inductive) and surface (capacitive) cells, respectively.

Following the standard Galerkin's testing procedure, topological elements, namely nodes and branches are generated and electrical lumped elements are identified modeling both the magnetic and electric field coupling.

Conductors are modeled by their ohmic resistance, while dielectrics requires modeling the excess charge due to the dielectric polarization [29]. Magnetic and electric field coupling are modeled by partial inductances and coefficients of potential, respectively.

The magnetic field coupling between two inductive volume cells $\alpha$ and $\beta$ is described by the partial inductance

$$
L p_{\alpha \beta}=\frac{\mu}{4 \pi} \frac{1}{a_{\alpha} a_{\beta}} \int_{u_{\alpha}} \int_{u_{\beta}} \frac{1}{R_{\alpha \beta}} d u_{\alpha} d u_{\beta}
$$

where $R_{\alpha \beta}$ is the distance between any two points in volumes $u_{\alpha}$ and $u_{\beta}$ with $a_{\alpha}$ and $a_{\beta}$ their cross sections. The electric field coupling between two capacitive surface cells $\delta$ and $\gamma$ is modeled by the coefficient of potential

$$
P_{\delta \gamma}=\frac{1}{4 \pi \varepsilon} \frac{1}{S_{\delta} S_{\gamma}} \int_{S_{\delta}} \int_{S \gamma} \frac{1}{R_{\delta \gamma}} d S_{\delta} d S_{\gamma}
$$

where $R_{\delta \gamma}$ is the distance between any two points on surfaces $\delta$ and $\gamma$, while $S_{\delta}$ and $S_{\gamma}$ denote the area of their respective surfaces.

Generalized Kirchoff's laws, for conductors, can be rewritten as

$$
\begin{aligned}
& \mathbf{P}^{-1} \frac{d \mathbf{v}(t)}{d t}-\mathbf{A}^{T} \mathbf{i}(t)+\mathbf{i}_{e}(t)=0 \\
& -\mathbf{A v}(t)-\mathbf{L}_{p} \frac{d \mathbf{i}(t)}{d t}-\mathbf{R i}(t)=\mathbf{0}
\end{aligned}
$$

where $\mathbf{A}$ is the connectivity matrix, $\mathbf{v}(t)$ denotes the node potentials to infinity, $\mathbf{i}(t)$ and $\mathbf{i}_{e}(t)$ represent the currents flowing in volume cells and the external currents, respectively.

When dielectrics are considered, the resistance voltage drop $\mathbf{R i}(t)$ is substituted by the excess capacitance voltage drop that is related to the excess charge by $\mathbf{v}_{d}(t)=\mathbf{C}_{d}^{-1} \mathbf{q}_{d}(t)$ [29]. Hence, for dielectric elementary cells, equations (3) become

$$
\begin{aligned}
& \mathbf{P}^{-1} \frac{d \mathbf{v}(t)}{d t}-\mathbf{A}^{T} \mathbf{i}(t)+\mathbf{i}_{e}(t)=0 \\
& -\mathbf{A v}(t)-\mathbf{L}_{p} \frac{d \mathbf{i}(t)}{d t}-\mathbf{v}_{d}(t)=\mathbf{0} \\
& \mathbf{i}(t)=\mathbf{C}_{d} \frac{d \mathbf{v}_{d}(t)}{d t}
\end{aligned}
$$

A selection matrix $\mathbf{K}$ is introduced to define the port voltages by selecting node potentials. The same matrix is used to obtain the external currents $\mathbf{i}_{e}(t)$ by the currents $\mathbf{i}_{s}(t)$ which are of opposite sign with respect to the $n_{p}$ port currents $\mathbf{i}_{p}(t)$

$$
\begin{aligned}
\mathbf{v}_{p}(t) & =\mathbf{K} \mathbf{v}(t) \\
\mathbf{i}_{e}(t) & =\mathbf{K}^{T} \mathbf{i}_{s}(t) .
\end{aligned}
$$

An example of PEEC circuit electrical quantities for a conductor elementary cell is illustrated, in the Laplace domain, 
in Fig. 1 where the current controlled voltage sources $s L_{p, i j} I_{j}$ and the current controlled current sources $I_{c c i}$ model the magnetic and electric field coupling, respectively

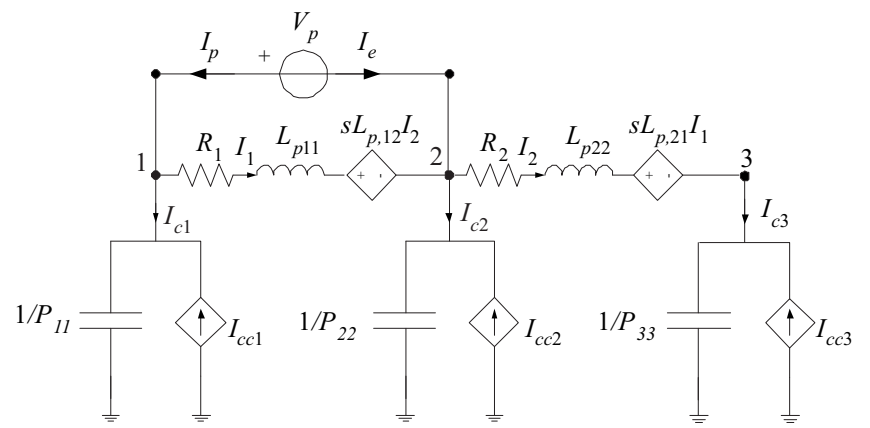

Fig. 1. Illustration of PEEC circuit electrical quantities for a conductor elementary cell.

\section{A. Descriptor representation of PEEC circuits}

Let us assume that the system under analysis consists of conductors and dielectrics. Let the current and charge density be defined in volumes and surface of conductors and dielectrics, respectively. The Galerkin's approach is applied to convert the continuous electromagnetic problem described by the EFIE to a discrete problem in terms of electrical circuit quantities, e.g. currents $\mathbf{i}(t)$ and node potentials $\mathbf{v}(t)$. Let us denote with $n_{n}$ the number of nodes and $n_{i}$ the number of branches where currents flow. Among the latter, we denote with $n_{c}$ and $n_{d}$ the number of branches of conductors and dielectrics, respectively. Furthermore, let us assume to be interested in generating an admittance representation having $n_{p}$ output currents $\mathbf{i}_{p}(t)$ under voltage excitation $\mathbf{v}_{p}(t)$. Since dielectrics require the excess capacitance to model the polarization charge [30], additional $n_{d}$ unknowns are needed in addition to currents. Hence, if the MNA approach [31] is used, the global number of unknowns is $n_{u}=n_{i}+n_{d}+n_{n}+n_{p}$. In a matrix form, the previous equations (3)-(5) read

$$
\begin{aligned}
& \underbrace{\left[\begin{array}{cccc}
\mathbf{I}_{n_{n}, n_{n}} & \mathbf{0}_{n_{n}, n_{i}} & \mathbf{0}_{n_{n}, n_{d}} & \mathbf{0}_{n_{n}, n_{p}} \\
\mathbf{0}_{n_{i}, n_{n}} & \mathbf{L}_{p} & \mathbf{0}_{n_{i}, n_{d}} & \mathbf{0}_{n_{i}, n_{p}} \\
\mathbf{0}_{n_{d}, n_{n}} & \mathbf{0}_{n_{d}, n_{i}} & \mathbf{C}_{d} & \mathbf{0}_{n_{d}, n_{p}} \\
\mathbf{0}_{n_{p}, n_{n}} & \mathbf{0}_{n_{p}, n_{i}} & \mathbf{0}_{n_{p}, n_{d}} & \mathbf{0}_{n_{p}, n_{p}}
\end{array}\right]}_{\mathbf{C}} \frac{d}{d t} \underbrace{\left[\begin{array}{c}
\mathbf{v}(t) \\
\mathbf{i}(t) \\
\mathbf{v}_{d}(t) \\
\mathbf{i}_{s}(t)
\end{array}\right]}_{\mathbf{x}(t)}= \\
& -\underbrace{\left[\begin{array}{cccc}
\mathbf{0}_{n_{n}, n_{n}} & -\mathbf{P A}^{T} & \mathbf{0}_{n_{n}, n_{d}} & \mathbf{P K}^{T} \\
\mathbf{A} & \mathbf{R} & \mathbf{\Phi} & \mathbf{0}_{n_{i}, n_{p}} \\
\mathbf{0}_{n_{d}, n_{n}} & -\boldsymbol{\Phi}^{T} & \mathbf{0}_{n_{d}, n_{d}} & \mathbf{0}_{n_{d}, n_{p}} \\
-\mathbf{K} & \mathbf{0}_{n_{p}, n_{i}} & \mathbf{0}_{n_{p}, n_{d}} & \mathbf{0}_{n_{p}, n_{p}}
\end{array}\right]}_{\mathbf{G}} \cdot \underbrace{\left[\begin{array}{c}
\mathbf{v}(t) \\
\mathbf{i}(t) \\
\mathbf{v}_{d}(t) \\
\mathbf{i}_{s}(t)
\end{array}\right]}_{\mathbf{x}(t)}+ \\
& \underbrace{\left[\begin{array}{c}
\mathbf{0}_{n_{n}+n_{i}+n_{d}, n_{p}} \\
-\mathbf{I}_{n_{p}, n_{p}}
\end{array}\right]}_{\mathbf{B}} \cdot \underbrace{\left[\mathbf{v}_{p}(t)\right]}_{\mathbf{u}(t)}
\end{aligned}
$$

where $\mathbf{I}_{n_{p}, n_{p}}$ is the identity matrix of dimensions equal to the number of ports. Matrix $\boldsymbol{\Phi}$ is

$$
\boldsymbol{\Phi}=\left[\begin{array}{l}
\mathbf{0}_{n_{c}, n_{d}} \\
\mathbf{I}_{n_{d}, n_{d}}
\end{array}\right]
$$

TABLE I

SCALED UNITS

\begin{tabular}{|c|c|}
\hline Voltage & $\mathrm{V}$ \\
Current & $\mathrm{mA}$ \\
Charge & $\mathrm{pC}$ \\
$P$ & $\mathrm{pF}^{-1}$ \\
$C_{d}$ & $\mathrm{pF}$ \\
$R$ & $\mathrm{k} \Omega$ \\
$L_{p}$ & $\mu \mathrm{H}$ \\
$f$ & $\mathrm{GHz}$ \\
$s$ & $\mathrm{~ns}$ \\
\hline
\end{tabular}

Then, potentials $\mathbf{v}(t)$ are expressed in terms of charges as

$$
\mathbf{v}(t)=\mathbf{P q}(t)
$$

Hence, equation (6) can be recast as

$$
\begin{aligned}
& \underbrace{\left[\begin{array}{cccc}
\mathbf{P} & \mathbf{0}_{n_{n}, n_{i}} & \mathbf{0}_{n_{n}, n_{d}} & \mathbf{0}_{n_{n}, n_{p}} \\
\mathbf{0}_{n_{i}, n_{n}} & \mathbf{L}_{p} & \mathbf{0}_{n_{i}, n_{d}} & \mathbf{0}_{n_{i}, n_{p}} \\
\mathbf{0}_{n_{d}, n_{n}} & \mathbf{0}_{n_{d}, n_{i}} & \mathbf{C}_{d} & \mathbf{0}_{n_{d}, n_{p}} \\
\mathbf{0}_{n_{p}, n_{n}} & \mathbf{0}_{n_{p}, n_{i}} & \mathbf{0}_{n_{p}, n_{d}} & \mathbf{0}_{n_{p}, n_{p}}
\end{array}\right]}_{\mathbf{C}} d \underbrace{\left[\begin{array}{c}
\mathbf{q}(t) \\
\mathbf{i}(t) \\
\mathbf{v}_{d}(t) \\
\mathbf{i}_{s}(t)
\end{array}\right]}_{\mathbf{x}(t)}= \\
& -\underbrace{\left[\begin{array}{cccc}
\mathbf{0}_{n_{n}, n_{n}} & -\mathbf{P A}^{T} & \mathbf{0}_{n_{n}, n_{d}} & \mathbf{P K}^{T} \\
\mathbf{A P} & \mathbf{R} & \mathbf{\Phi} & \mathbf{0}_{n_{i}, n_{p}} \\
\mathbf{0}_{n_{d}, n_{n}} & -\boldsymbol{\Phi}^{T} & \mathbf{0}_{n_{d}, n_{d}} & \mathbf{0}_{n_{d}, n_{p}} \\
-\mathbf{K P} & \mathbf{0}_{n_{p}, n_{i}} & \mathbf{0}_{n_{p}, n_{d}} & \mathbf{0}_{n_{p}, n_{p}}
\end{array}\right]}_{\mathbf{G}} \cdot \underbrace{\left[\begin{array}{c}
\mathbf{q}(t) \\
\mathbf{i}(t) \\
\mathbf{v}_{d}(t) \\
\mathbf{i}_{s}(t)
\end{array}\right]}_{\mathbf{x}(t)}+ \\
& \underbrace{\left[\begin{array}{c}
\mathbf{0}_{n_{n}+n_{i}+n_{d}, n_{p}} \\
-\mathbf{I}_{n_{p}, n_{p}}
\end{array}\right]}_{\mathbf{B}} \cdot \underbrace{\left[\mathbf{v}_{p}(t)\right]}_{\mathbf{u}(t)}
\end{aligned}
$$

In a more compact form, the previous equations (9) can be rewritten as

$$
\begin{aligned}
\mathbf{C} \frac{d \mathbf{x}(t)}{d t} & =-\mathbf{G x}(t)+\mathbf{B u}(t) \\
\mathbf{i}_{p}(t) & =\mathbf{L}^{T} \mathbf{x}(t)
\end{aligned}
$$

where $\mathbf{x}(t)=\left[\begin{array}{llll}\mathbf{q}(t) & \mathbf{i}(t) & \mathbf{v}_{d}(t) & \mathbf{i}_{s}(t)\end{array}\right]^{T}$. Since this is an $n_{p}$-port formulation, whereby the only sources are the voltage sources at the $n_{p}$-port nodes, $\mathbf{B}=\mathbf{L}$ where $\mathbf{B} \in \Re^{n_{u} \times n_{p}}$.

\section{B. Scaling}

The system of equations (9) is typically ill-conditioned because charges are usually much smaller than currents and voltages. Correspondingly, the entries of the matrix $\mathbf{P}$ are larger than other elements in matrices $\mathbf{C}$ and $\mathbf{G}$ by several orders of magnitude. The ill-conditioning of (9) prevents MOR methods to be efficiently applied. In order to mitigate such a problem, scaling can be adopted. The units of the electrical quantities are changed consistently as shown in Table I.

\section{Properties PEEC formulation}

In order to apply the proposed PMOR technique, it is important to specify the properties of the matrices involved in the PEEC formulation (9).

Both matrices describing electric and magnetic field coupling, $\mathbf{P}$ and $\mathbf{L}_{p}$ respectively, are full symmetric matrices. In 
the case of orthogonal geometries, mutual partial inductances corresponding to orthogonal currents are equal to zero. Even in this case, rows and columns can be recast so that the partial inductance matrix $\mathbf{L}_{p}$ is block-diagonal. Since each block is symmetric positive definite, the overall matrix $\mathbf{L}_{p}$ is symmetric positive definite as well. The coefficient of potential matrix $\boldsymbol{P}$ is also symmetric positive definite [32].

When pulse basis functions are used, as is in the standard PEEC formulation [2], resistance and excess capacitance matrices, $\mathbf{R}$ and $\mathbf{C}_{d}$ respectively, are diagonal and symmetric positive semidefinite and definite. The matrix $\mathbf{R}$ is diagonal, with positive diagonal elements corresponding to conductor elementary cells, while the diagonal elements corresponding to dielectric elementary cells are equal to zero. The matrix $\mathbf{C}_{d}$ is diagonal with all the diagonal elements positive.

Assuming the previous matrix properties, it is easy to prove that the matrices $\mathbf{C}, \mathbf{G}$ satisfy the following properties

$$
\begin{aligned}
& \mathbf{C}=\mathbf{C}^{T} \geq 0 \\
& \mathbf{G}+\mathbf{G}^{T} \geq 0
\end{aligned}
$$

The properties of the PEEC matrices $\mathbf{B}=\mathbf{L}, \mathbf{C}=\mathbf{C}^{T} \geq$ $0, \mathbf{G}+\mathbf{G}^{T} \geq 0$ ensure the passivity of the PEEC admittance model $\mathbf{Y}(s)=\mathbf{L}^{T}(s \mathbf{C}+\mathbf{G})^{-1} \mathbf{B}$ [33] and allow to exploit the passivity-preserving capability of the Laguerre-SVD MOR algorithm [19]. When performing transient analysis, stability and passivity must be guaranteed. It is known that, while a passive system is also stable, the reverse is not necessarily true [34], which is crucial when the reduced model is to be utilized in a general-purpose analysis-oriented nonlinear simulator (e.g. SPICE). Passivity refers to the property of systems that cannot generate more energy than they absorb through their electrical ports. When the system is terminated on any arbitrary passive loads, none of them will cause the system to become unstable [35], [36].

\section{PARAMETERIZED MOdel ORder REDUCTION}

In this section we describe a PMOR algorithm that is able to include, in addition to frequency, $\mathrm{N}$ design parameters $\boldsymbol{g}=\left(g^{(1)}, \ldots, g^{(\mathrm{N})}\right)$ in a parameterized ROM, such as the layout features of a circuit (e.g. lengths, widths,...) or the substrate parameters (e.g. thickness, dielectric permittivity, losses,...). The main objective of the PMOR method is to accurately approximate the original scalable system (having a high complexity) with a reduced scalable system (having a low complexity) by capturing the behavior of the original system with respect to frequency and other design parameters. The design space $\mathcal{D}(\boldsymbol{g})$ is considered as the parameter space $\mathcal{P}(s, \boldsymbol{g})$ without frequency. The parameter space $\mathcal{P}(s, \boldsymbol{g})$ contains all parameters $(s, \boldsymbol{g})$. If the parameter space is $(\mathrm{N}+1)$ dimensional, the design space is $\mathrm{N}$-dimensional. The proposed algorithm guarantees stability and passivity of a parameterized ROM over the entire design space of interest. Two data grids are used in the modeling process: an estimation grid and a validation grid. The first grid is utilized to build parameterized ROMs, while the second grid, more dense than the previous one, is utilized to assess the capability of parameterized ROMs of describing the system under study in points of the design space previously not used for its construction. To clarify the use of these two design space grids, we show in Fig. 2 a possible estimation and validation design space grid in the case of two design parameters $\boldsymbol{g}=\left(g^{(1)}, g^{(2)}\right)$.

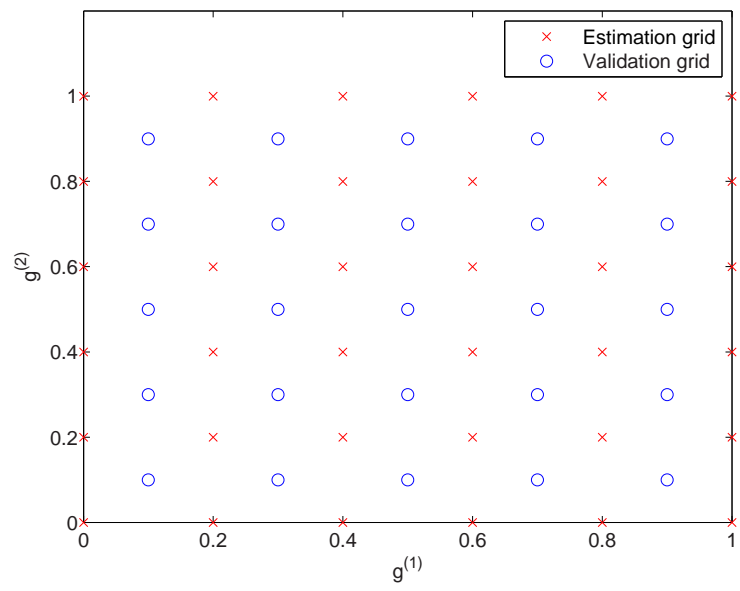

Fig. 2. An example of estimation and validation design space grid.

\section{A. PMOR algorithm}

Considering the influence of the design parameters $\boldsymbol{g}=$ $\left(g^{(1)}, \ldots, g^{(\mathrm{N})}\right)$, the MNA formulation (10a)-(10b) becomes

$$
\begin{aligned}
\mathbf{C}(\boldsymbol{g}) \frac{d \mathbf{x}(t, \boldsymbol{g})}{d t} & =-\mathbf{G}(\boldsymbol{g}) \mathbf{x}(t, \boldsymbol{g})+\mathbf{B u}(t) \\
\mathbf{i}_{p}(t, \boldsymbol{g}) & =\mathbf{L}^{T} \mathbf{x}(t, \boldsymbol{g})
\end{aligned}
$$

We assume that a topologically fixed discretisation mesh is used and it is independent from the specific design parameters values. It preserves the size of the system matrices as well as the numbering of the mesh nodes and mesh edges. The mesh is only locally stretched or shrunk, when shape parameters are modified. In general, the global coordinates of the nodes as well as the length and orientation of the edges of the topologically fixed mesh change when shape parameters change; however, these changes are neither introducing new state variables nor eliminating existing state variables. The matrices $\mathbf{B}, \mathbf{L}^{T}$ are uniquely determined by the circuit topology and therefore remain constant, while the matrices $\mathbf{C}$ and $\mathbf{G}$ are defined as functions of the design parameters. At a deeper level in the MNA equations (12a)-(12b), the previous assumptions lead to have $\mathbf{P}(\boldsymbol{g}), \mathbf{L}_{p}(\boldsymbol{g}), \mathbf{C}_{d}(\boldsymbol{g}), \mathbf{R}(\boldsymbol{g})$, while the other internal PEEC matrices $\mathbf{A}, \mathbf{\Phi}, \mathbf{K}$ are constant. The proposed PMOR method starts from computing the multivariate models $\overline{\mathbf{P}}(\boldsymbol{g}), \overline{\mathbf{L}}_{p}(\boldsymbol{g}), \overline{\mathbf{C}}_{d}(\boldsymbol{g}), \overline{\mathbf{R}}(\boldsymbol{g})$ guaranteeing some matrix properties, as explained in Section III-B.

When the multivariate models $\overline{\mathbf{P}}(\boldsymbol{g}), \overline{\mathbf{L}}_{p}(\boldsymbol{g}), \overline{\mathbf{C}}_{d}(\boldsymbol{g}), \overline{\mathbf{R}}(\boldsymbol{g})$ are computed, instead of assembling a PEEC model and performing a MOR step for each point of interest $\widehat{\boldsymbol{g}}=$ $\left(g_{k_{1}}^{(1)}, \ldots, g_{k_{N}}^{(\mathrm{N})}\right)$ in the design space, the Laguerre-SVD MOR method [19] is applied to each PEEC model related to the estimation design space grid 
- choose a value for $\alpha$ (positive scaling parameter of Laguerre basis functions) and the reduced order $q$;

- solve $(\mathbf{G}+\alpha \mathbf{C}) \mathbf{Q}_{0}=\mathbf{B}$;

- for $k=1, \ldots, q-1$ solve $(\mathbf{G}+\alpha \mathbf{C}) \mathbf{Q}_{k}=(\mathbf{G}-$ $\alpha \mathbf{C}) \mathbf{Q}_{k-1}$

- $\mathbf{K}_{r}=\left[\mathbf{Q}_{0}, \ldots, \mathbf{Q}_{q-1}\right]$;

and a corresponding set of Krylov matrices $\mathbf{K}_{r}$ is computed. Then, this set of Krylov matrices is interpolated and modeled as $\overline{\mathbf{K}}_{r}(\mathbf{g})$. The sampling density in the estimation design space grid is important to accurately describe the parameterized behavior of an EM system under study over the entire design space of interest. A technique to choose the number of points in the estimation grid can be found in [37]. Once the multivariate models $\overline{\mathbf{P}}(\boldsymbol{g}), \overline{\mathbf{L}}_{p}(\boldsymbol{g}), \overline{\mathbf{C}}_{d}(\boldsymbol{g}), \overline{\mathbf{R}}(\boldsymbol{g}), \overline{\mathbf{K}}_{r}(\boldsymbol{g})$ are built, a PEEC model $\mathbf{Y}(s, \widehat{\boldsymbol{g}})=\mathbf{L}^{T}(s \overline{\mathbf{C}}(\widehat{\boldsymbol{g}})+\overline{\mathbf{G}}(\widehat{\boldsymbol{g}}))^{-1} \mathbf{B}$ can be assembled and a projection matrix $\mathbf{U}(\widehat{\boldsymbol{g}})$ can be computed by means of the singular value decomposition [38] of $\overline{\mathbf{K}}_{r}(\widehat{\boldsymbol{g}})$ [19] for any point $\widehat{\boldsymbol{g}}=\left(g_{k_{1}}^{(1)}, \ldots, g_{k_{N}}^{(\mathrm{N})}\right)$

$$
\mathbf{U}(\widehat{\boldsymbol{g}}) \boldsymbol{\Sigma}(\widehat{\boldsymbol{g}}) \mathbf{V}(\widehat{\boldsymbol{g}})^{T}=\operatorname{SVD}\left[\overline{\mathbf{K}}_{r}(\widehat{\boldsymbol{g}})\right]
$$

Finally, a congruence transformation is applied on $\mathbf{C}(\widehat{\boldsymbol{g}}), \mathbf{G}(\widehat{\boldsymbol{g}}), \mathbf{L}, \mathbf{B}$ using $\mathbf{U}(\widehat{\boldsymbol{g}})$ [19]

$$
\begin{aligned}
\mathbf{C}_{r}(\widehat{\boldsymbol{g}}) & =\mathbf{U}(\widehat{\boldsymbol{g}})^{T} \mathbf{C}(\widehat{\boldsymbol{g}}) \mathbf{U}(\widehat{\boldsymbol{g}}) \\
\mathbf{G}_{r}(\widehat{\boldsymbol{g}}) & =\mathbf{U}(\widehat{\boldsymbol{g}})^{T} \mathbf{G}(\widehat{\boldsymbol{g}}) \mathbf{U}(\widehat{\boldsymbol{g}}) \\
\mathbf{B}_{r}(\widehat{\boldsymbol{g}}) & =\mathbf{U}(\widehat{\boldsymbol{g}})^{T} \mathbf{B} \\
\mathbf{L}_{r}(\widehat{\boldsymbol{g}}) & =\mathbf{U}(\widehat{\boldsymbol{g}})^{T} \mathbf{L}
\end{aligned}
$$

to obtain the parameterized reduced model. A flowchart that describes the different steps of the proposed PMOR method is shown in Fig. 3. Concerning the reduced order, which represents the column dimension of $\overline{\mathbf{K}}_{r}(\boldsymbol{g})$ and $\mathbf{U}(\boldsymbol{g})$, it is chosen by a bottom-up approach: it is increased as long as a certain RMS-error threshold is satisfied in the validation design space grid.

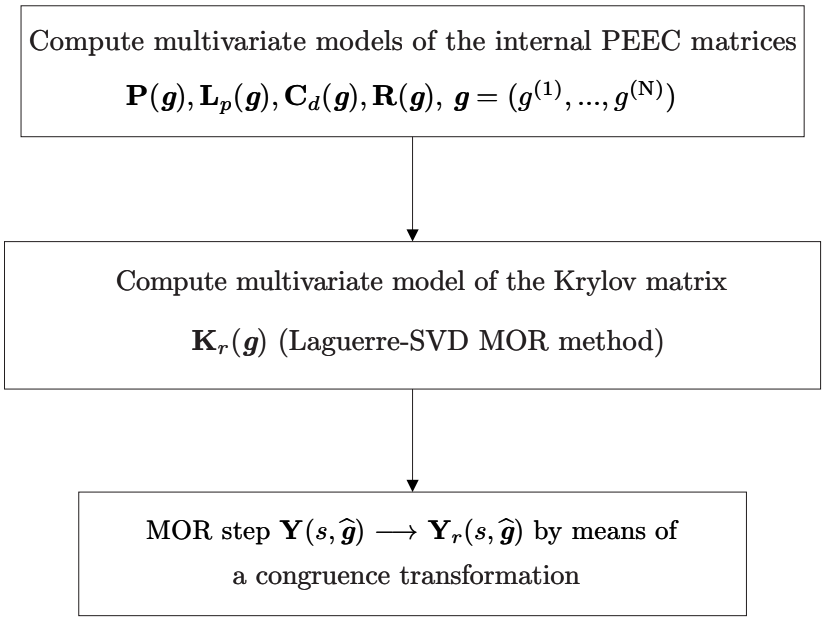

Fig. 3. Flowchart of the proposed PMOR method.

\section{B. Multivariate interpolation of the internal PEEC matrices}

Starting from multivariate data samples $\left\{\boldsymbol{g}_{k}, \mathbf{P}\left(\boldsymbol{g}_{k}\right), \mathbf{L}_{p}\left(\boldsymbol{g}_{k}\right), \mathbf{C}_{d}\left(\boldsymbol{g}_{k}\right), \mathbf{R}\left(\boldsymbol{g}_{k}\right), \mathbf{K}_{r}\left(\boldsymbol{g}_{k}\right)\right\}_{k=1}^{K t o t}, \quad$ the multivariate models $\overline{\mathbf{P}}(\boldsymbol{g}), \overline{\mathbf{L}}_{p}(\boldsymbol{g}), \overline{\mathbf{C}}_{d}(\boldsymbol{g}), \overline{\mathbf{R}}(\boldsymbol{g}), \overline{\mathbf{K}}_{\mathbf{r}}(\boldsymbol{g})$ are built. While the interpolation process of the set of Krylov matrices is performed without any constraint, the multivariate models of the internal PEEC matrices preserve the positive definiteness of $\mathbf{P}(\boldsymbol{g}), \mathbf{L}_{p}(\boldsymbol{g}), \mathbf{C}_{d}(\boldsymbol{g})$ and the positive semidefiniteness of $\mathbf{R}(\boldsymbol{g})$. Consequently, the properties (11a)-(11b) of an admittance PEEC model and its related passivity are satisfied for any point $\widehat{\boldsymbol{g}}=\left(g_{k_{1}}^{(1)}, \ldots, g_{k_{N}}^{(\mathrm{N})}\right)$ over the design space. Since the matrices $\mathbf{R}(\boldsymbol{g})$ and $\mathbf{C}_{d}(\boldsymbol{g})$ are diagonal, only the diagonal elements are interpolated by means of a positivity-preserving interpolation scheme. Multivariate interpolation schemes that belong to a class of positive interpolation operators [39] can be used, e.g. Shepard's method [40], multilinear and simplicial methods [41]. Such interpolation schemes have interpolation kernel functions that only depend on the design space grid points. In the case of multilinear interpolation, each interpolated matrix $\mathbf{T}\left(g^{(1)}, \ldots, g^{(N)}\right)$, being in turn $\mathbf{C}_{d}(\boldsymbol{g}), \mathbf{R}(\boldsymbol{g})$, can be written as

$$
\begin{aligned}
& \mathbf{T}\left(g^{(1)}, \ldots, g^{(N)}\right)= \\
& =\sum_{k_{1}=1}^{K_{1}} \cdots \sum_{k_{N}=1}^{K_{N}} \mathbf{T}_{\left(g_{k_{1}}^{(1)}, \ldots, g_{k_{N}}^{(N)}\right)} \ell_{k_{1}}\left(g^{(1)}\right) \cdots \ell_{k_{N}}\left(g^{(N)}\right)
\end{aligned}
$$

where $\quad \mathbf{T}_{\left(g_{k_{1}}^{(1)}, \ldots, g_{k_{N}}^{(N)}\right)} \quad$ are $\quad$ in turn $\mathbf{C}_{d,\left(g_{k_{1}}^{(1)}, \ldots, g_{k_{N}}^{(N)}\right)}, \mathbf{R}_{\left(g_{k_{1}}, \ldots, g_{k_{N}}^{(N)}\right)}^{(1)}$, therefore the discrete set of $\mathbf{C}_{d}, \mathbf{R}$ matrices related to the estimation design space grid. Each interpolation kernel $\ell_{k_{i}}\left(g^{(i)}\right), i=1, \ldots, N$ is selected as in piecewise linear interpolation

$$
\begin{aligned}
& \frac{g^{(i)}-g_{k_{i}-1}^{(i)}}{g_{k_{i}}^{(i)}-g_{k_{i}-1}^{(i)}}, g^{(i)} \in\left[g_{k_{i}-1}^{(i)}, g_{k_{i}}^{(i)}\right], k_{i}=2, \ldots, K_{i}, \\
& \frac{g_{k_{i}+1}^{(i)}-g^{(i)}}{g_{k_{i}+1}^{(i)}-g_{k_{i}}^{(i)}}, g^{(i)} \in\left[g_{k_{i}}^{(i)}, g_{k_{i}+1}^{(i)}\right], k_{i}=1, \ldots, K_{i}-1,
\end{aligned}
$$

0 , otherwise

Hence, the interpolation kernels $\ell_{k_{i}}\left(g^{(i)}\right), i=1, \ldots, N$ are independent from the matrices used in the interpolation process and depend only on the design space grid points. Other interpolation schemes have kernel functions that depend on the matrices used in the interpolation process, e.g. multivariate cubic spline interpolation [42]. It is a useful technique to interpolate multivariate data points due to its stable and smooth characteristics and it performs elementwise interpolation. Unfortunately, although ordinary spline schemes are generally well behaved, they do not prevent overshoot and undesired oscillations at intermediate points, that can violate inherited data features as positivity. Some modified spline interpolation 
schemes that are able to preserve positivity of the data samples in the univariate case are described in [43]-[45]. Another simpler and straightforward approach to preserve positivity using the ordinary splines is proposed in this paper and it is composed of three steps: 1) an analytical mapping of the data samples is performed, 2) the new data samples are interpolated using ordinary splines without any constraint, 3) the interpolated data samples are transformed back by the inverse mapping. The mapping function has to be able to ensure the positivity of the interpolated data samples after the inverse mapping. For any positive diagonal matrix entry $f(\boldsymbol{g})$ of the matrices $\mathbf{C}_{d}(\boldsymbol{g}), \mathbf{R}(\boldsymbol{g})$ under modeling, the following mapping function is used

$$
M(\boldsymbol{g})=\log \left(\frac{f(\boldsymbol{g})}{\min (f(\boldsymbol{g}))}\right), \boldsymbol{g} \in\left\{\boldsymbol{g}_{k}\right\}_{k=1}^{K_{t o t}}
$$

Once the transformed data samples are modeled by using multivariate splines, the inverse mapping function

$$
M_{i n v}(\boldsymbol{g})=\min (f(\boldsymbol{g})) \exp (M(\boldsymbol{g})), \boldsymbol{g} \in\left\{\boldsymbol{g}_{k}\right\}_{k=1}^{K_{\text {tot }, \text { interp }}}
$$

is used for the back transformation. It is straightforward to verify that the following procedure ensure the positivity of the final interpolated values. While a diagonal matrix is positive definite if and only if all the diagonal elements are positive, a non-diagonal matrix requires more general conditions. The matrix $\mathbf{P}(\boldsymbol{g})$ is full, symmetric and positive definite, while the matrix $\mathbf{L}_{p}(\boldsymbol{g})$ is symmetric, positive definite and in general a certain degree of sparsity can be present, due to orthogonal elementary cells. It is easy to show that multivariate interpolation schemes that belong to a class of positive interpolation operators [39]-[41] are able to preserve the positive definiteness property, when they are applied to positive definite matrices. When the interpolation of positive definite non-diagonal matrices is performed elementwise by schemes with kernel functions that depend on the matrices used in the interpolation process (e.g. multivariate cubic spline interpolation), the following procedure can be used to guarantee the positive definiteness property. Let us denote

$$
\mathcal{S}(\mathrm{R})=\left\{\mathbf{Q} \in \mathcal{M}(\mathrm{R}), \mathbf{Q}^{T}=\mathbf{Q}\right\}
$$

the space of all $\mathrm{R} \times \mathrm{R}$ real symmetric matrices with $\mathcal{M}(\mathrm{R})$ the space of $R \times R$ real matrices and

$$
\mathcal{P}(\mathrm{R})=\{\mathbf{Q} \in \mathcal{S}(\mathrm{R}), \mathbf{Q}>0\}
$$

the space of all $\mathrm{R} \times \mathrm{R}$ real symmetric positive-definite matrices. It is well known that the matrix exponential is a one-to-one map from $\mathcal{S}(\mathrm{R})$ to $\mathcal{P}(\mathrm{R})$. In other words, the matrix exponential of any real symmetric matrix is a real symmetric positivedefinite matrix, and the inverse of the matrix exponential (i.e., principal matrix logarithm) of any real symmetric positivedefinite matrix is a real symmetric matrix [46], [47]. Exploiting such property of the exponential map, the matrices $\mathbf{P}(\boldsymbol{g})$, $\mathbf{L}_{p}(\boldsymbol{g})$ that are symmetric and positive definite are mapped from $\mathcal{P}(\mathrm{R})$ to $\mathcal{S}(\mathrm{R})$ using the principal matrix logarithm operator, then only the lower or upper triangular part is interpolated elementwise using the ordinary splines. Finally, the matrices are mapped back by the matrix exponential operator which results in symmetric positive definite matrices, therefore the original properties of the matrices $\mathbf{P}(\boldsymbol{g}), \mathbf{L}_{p}(\boldsymbol{g})$ are preserved. We propose a multivariate interpolation process that is able to preserve the positive definiteness of $\mathbf{P}(\boldsymbol{g}), \mathbf{L}_{p}(\boldsymbol{g}), \mathbf{C}_{d}(\boldsymbol{g})$ and the positive semidefiniteness of $\mathbf{R}(\boldsymbol{g})$. Consequently, the properties (11a)-(11b) of the admittance PEEC model $\mathbf{Y}(s, \boldsymbol{g})=$ $\mathbf{L}^{T}(s \overline{\mathbf{C}}(\boldsymbol{g})+\overline{\mathbf{G}}(\boldsymbol{g}))^{-1} \mathbf{B}$ and its related passivity are satisfied for any point $\widehat{\boldsymbol{g}}=\left(g_{k_{1}}^{(1)}, \ldots, g_{k_{N}}^{(\mathrm{N})}\right)$ over the design space. The overall computational complexity of the presented PMOR algorithm can be divided into: 1) complexity of computing the multivariate models of $\overline{\mathbf{P}}(\boldsymbol{g}), \overline{\mathbf{L}}_{p}(\boldsymbol{g}), \overline{\mathbf{C}}_{d}(\boldsymbol{g}), \overline{\mathbf{R}}(\boldsymbol{g}), \overline{\mathbf{K}}_{r}(\boldsymbol{g})$ by interpolation, 2) complexity of the SVD operation on $\overline{\mathbf{K}}_{r}(\mathbf{g})$ to obtain the projection matrix $\mathbf{U}(\boldsymbol{g}), 3)$ complexity of the congruence transformation by means of $\mathbf{U}(\mathbf{g})$. Which step is the most computationally expensive cannot be established in advance, since the computational complexity of the interpolation process depends on the chosen interpolation scheme. Concerning the SVD operation, it can be replaced by cheaper modified Gram-Schmidt (MGS) and Householder QR (HQR) operations [19], [38], which are computationally cheaper.

\section{Passivity assessment considerations}

The properties of the PEEC matrices, the multivariate interpolation approach and the Laguerre-SVD MOR algorithm ensure overall stability and passivity for a parameterized ROM $\mathbf{Y}_{r}(s, \boldsymbol{g})$ by construction. Although no passivity check is required for $\mathbf{Y}_{r}(s, \boldsymbol{g})$, the authors describe in this section a passivity test for the sake of completeness. Let us assume that $\mathbf{Y}_{r}(s, \boldsymbol{g})$ is obtained and one wants to perform a passivity test for a specific point $\widehat{\boldsymbol{g}}$ in the design space. If the descriptor matrix $\mathbf{C}_{\mathbf{r}}$ of $\mathbf{Y}_{r}(s, \widehat{\boldsymbol{g}})$ is singular, the procedure described in [48] is used to convert the descriptor system into a standard state-space model

$$
\begin{aligned}
\frac{d \boldsymbol{x}(t)}{d t} & =\mathcal{A} \boldsymbol{x}(t)+\mathcal{B} \boldsymbol{u}(t) \\
\boldsymbol{y}(t) & =\mathcal{C} \boldsymbol{x}(t)+\mathcal{D} \boldsymbol{u}(t)
\end{aligned}
$$

otherwise the standard state-space model can be obtained by

$$
\begin{aligned}
\mathcal{A} & =-\mathbf{C}_{\mathbf{r}}{ }^{-1} \mathbf{G}_{\mathbf{r}} \\
\mathcal{B} & =\mathbf{C}_{\mathbf{r}}{ }^{-1} \mathbf{B}_{\mathbf{r}} \\
\mathcal{C} & =\mathbf{B}_{\mathbf{r}}{ }^{T} \\
\mathcal{D} & =\mathbf{D}_{\mathbf{r}}
\end{aligned}
$$

Once $\mathbf{Y}_{r}(s, \widehat{\boldsymbol{g}})$ is transformed into a standard state-space form, its passivity can be verified by computing the eigenvalues of an associated Hamiltonian matrix [49]

$$
\tilde{\mathcal{H}}=\left[\begin{array}{cc}
\mathcal{A}-\mathcal{B} \mathcal{R}^{-1} \mathcal{C} & \mathcal{B R}^{-1} \mathcal{B}^{T} \\
-\mathcal{C}^{T} \mathcal{R}^{-1} \mathcal{C} & -\mathcal{A}^{T}+\mathcal{C}^{T} \mathcal{R}^{-1} \mathcal{B}^{T}
\end{array}\right]
$$

with $\mathcal{R}=\mathcal{D}+\mathcal{D}^{T}$. The system $\mathbf{Y}_{r}(s, \widehat{\boldsymbol{g}})$ is passive if $\tilde{\mathcal{H}}$ has no purely imaginary eigenvalues. This passivity test can only be applied if $\mathcal{D}+\mathcal{D}^{T}$ is not singular. If such singularity exists, 
the modified Hamiltonian-based passivity check proposed in [50] should be used.

\section{NUMERICAL RESULTS}

This section presents two numerical examples that validate the proposed PMOR method. Let us define the weighted RMSerror as:

$$
\begin{aligned}
& \operatorname{Err}(\boldsymbol{g})= \\
& =\sqrt{\frac{\sum_{i=1}^{\left(N_{\text {port }}\right)^{2}} \sum_{k=1}^{K_{s}}\left|w_{Y_{i}}\left(s_{k}, \boldsymbol{g}\right)\left(Y_{r, i}\left(s_{k}, \boldsymbol{g}\right)-Y_{i}\left(s_{k}, \boldsymbol{g}\right)\right)\right|^{2}}{\left(N_{\text {port }}\right)^{2} K_{s}}}
\end{aligned}
$$

with

$$
w_{Y_{i}}(s, \boldsymbol{g})=\left|\left(Y_{i}(s, \boldsymbol{g})\right)^{-1}\right|
$$

where $N_{\text {port }}$ is the number of system ports and $K_{s}$ is the number of frequency samples. The worst case RMS-error over the validation grid is chosen to assess the accuracy and the quality of parameterized ROMs

$$
\begin{gathered}
\boldsymbol{g}_{\text {max }}=\underset{\boldsymbol{g}}{\operatorname{argmax}} \operatorname{Err}(\boldsymbol{g}), \boldsymbol{g} \in \text { validation grid } \\
\operatorname{Err}_{\max }=\operatorname{Err}\left(\boldsymbol{g}_{\max }\right)
\end{gathered}
$$

and it is used in the numerical examples. The proposed PMOR algorithm was implemented in Matlab R2009A [51] and all experiments were carried out on Windows platform on Intel Core2 Extreme CPU Q9300 2.53GHz machines with 8GB RAM.

\section{A. Two coupled microstrips with variable spacing}

Two coupled microstrips (length $L=2 \mathrm{~cm}$ ) have been modeled in this example. The cross section is shown in Fig. 4. The conductors have width $W=500 \mu \mathrm{m}$ and thickness $t=50 \mu \mathrm{m}$, the dielectric is $800 \mu \mathrm{m}$ thick. A bivariate ROM is built as a function of the spacing $S$ between the microstrips in addition to frequency. Their corresponding ranges are shown in Table II.

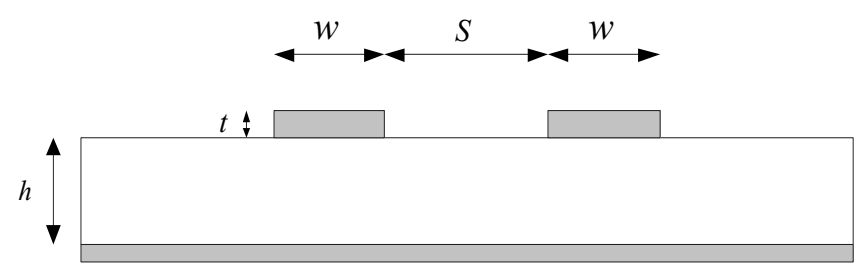

Fig. 4. Cross section of the coupled microstrips.

The PEEC method is used to compute the $\mathbf{C}, \mathbf{G}, \mathbf{B}, \mathbf{L}$ matrices in (10a)-(10b) for 25 values of the spacing. The order of all original PEEC models is equal to $n_{u}=2640$. The multivariate models $\overline{\mathbf{P}}(\boldsymbol{g}), \overline{\mathbf{L}}_{p}(\boldsymbol{g}), \overline{\mathbf{C}}_{d}(\boldsymbol{g}), \overline{\mathbf{R}}(\boldsymbol{g}), \overline{\mathbf{K}}_{r}(\boldsymbol{g})$ are computed by spline interpolation using only 9 spacing
TABLE II

PARAMETERS OF THE COUPLED MICROSTRIPS.

\begin{tabular}{|l|c|c|}
\hline Parameter & Min & Max \\
\hline Frequency $($ freq $)$ & $1 \mathrm{kHz}$ & $4 \mathrm{GHz}$ \\
Spacing $(S)$ & $1 \mathrm{~mm}$ & $4 \mathrm{~mm}$ \\
\hline
\end{tabular}

values and with a CPU time equal to $9.6 \mathrm{~s}$. Then, the bivariate $\operatorname{ROM} \mathbf{Y}_{r}(s, S)$ is obtained with a reduced order $q=38$.Fig. 5 shows the magnitude of the parameterized ROM of $\mathbf{Y}_{11}(s, S)$, while Fig. 6 compares the magnitude of $\mathbf{Y}_{11}(s, S), \mathbf{Y}_{12}(s, S)$ and their parameterized ROMs for the spacing values $S=\{1.125,2.375,3.875\} \mathrm{mm}$. These specific spacing values have not been used during the construction of the multivariate models $\overline{\mathbf{P}}(\boldsymbol{g}), \overline{\mathbf{L}}_{p}(\boldsymbol{g}), \overline{\mathbf{C}}_{d}(\boldsymbol{g}), \overline{\mathbf{R}}(\boldsymbol{g}), \overline{\mathbf{K}}_{r}(\boldsymbol{g})$, nevertheless an excellent agreement between model and data can be observed. The worst case RMS-error defined in (27) is equal to $1.8 \cdot 10^{-2}$ and it occurs for $g_{\max }=S=3.875 \mathrm{~mm}$. Fig. 7 shows the minimum absolute value of the real part of the Hamiltonian matrix eigenvalues over a dense sweep of the design space. Since there are no purely imaginary eigenvalues, the parameterized ROM is passive over the design space of interest. As clearly seen, the parameterized ROM captures the behavior of the system very accurately, while guaranteeing stability and passivity over the entire design space.

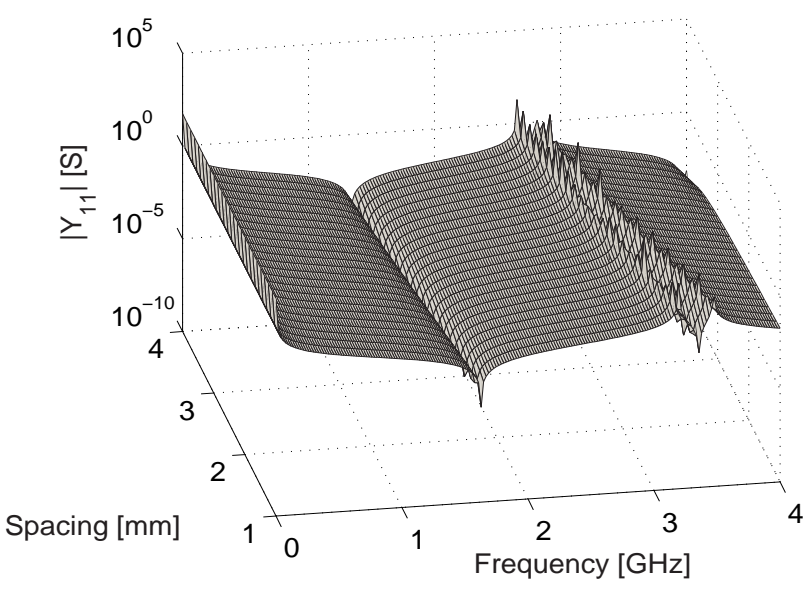

Fig. 5. Magnitude of the bivariate ROM of $\mathbf{Y}_{11}(s, S)$.

\section{B. Spiral inductor with variable horizontal and vertical length}

An integrated spiral inductor has been modeled in this example. The structure is shown in Fig. 8. The conductors width is equal to $46 \mu \mathrm{m}$. A trivariate ROM is built as a function of the horizontal $L_{x}$ and vertical $L_{y}$ length of the spiral inductor in addition to frequency. Their corresponding ranges are shown in Table III.

The PEEC method is used to compute the $\mathbf{C}, \mathbf{G}, \mathbf{B}, \mathbf{L}$ matrices in (10a)-(10b) for 11 values of $L_{x}$ and 11 values of $L_{y}$. The order of all original PEEC models is equal to $n_{u}=801$. The multivariate models $\overline{\mathbf{P}}(\boldsymbol{g}), \overline{\mathbf{L}}_{p}(\boldsymbol{g}), \overline{\mathbf{C}}_{d}(\boldsymbol{g}), \overline{\mathbf{R}}(\boldsymbol{g}), \overline{\mathbf{K}}_{r}(\boldsymbol{g})$ are computed by spline interpolation using only 6 values 

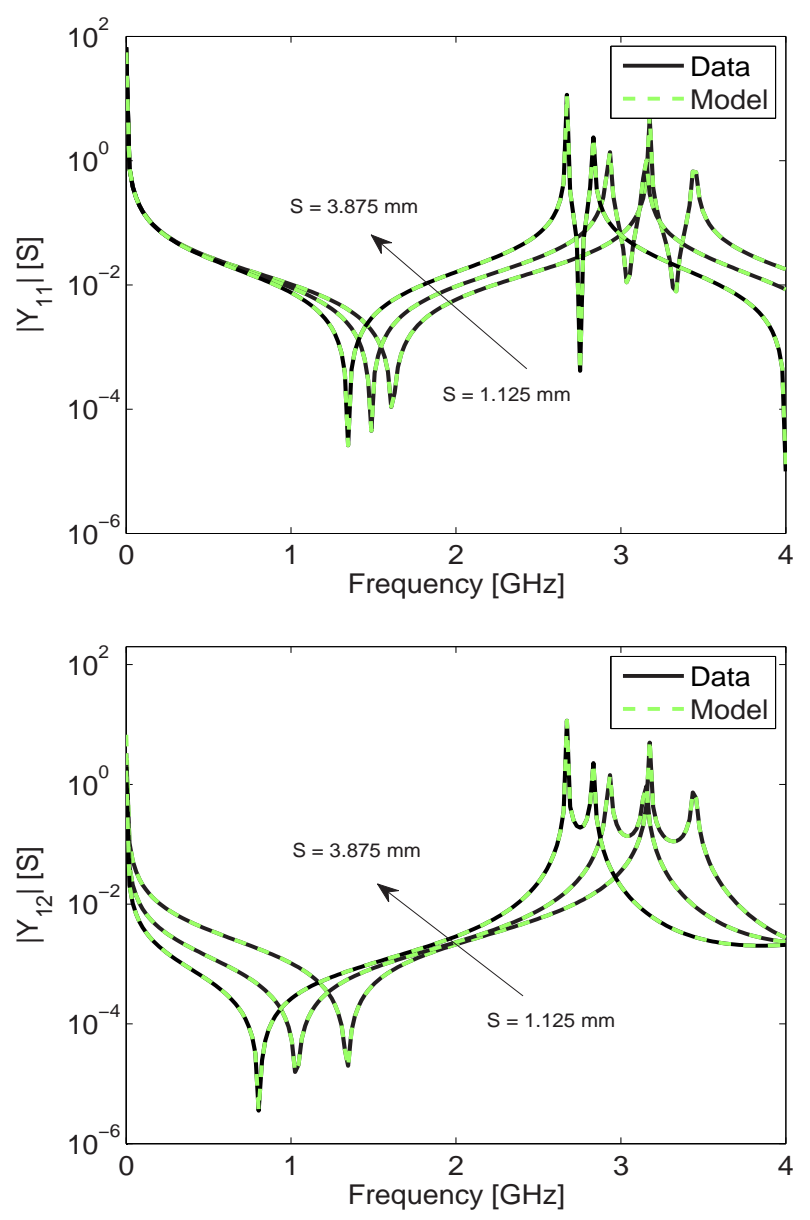

Fig. 6. Magnitude of the bivariate ROMs of $\mathbf{Y}_{11}(s, S)$ and $\mathbf{Y}_{12}(s, S)$ $(S=\{1.125,2.375,3.875\} \mathrm{mm})$.

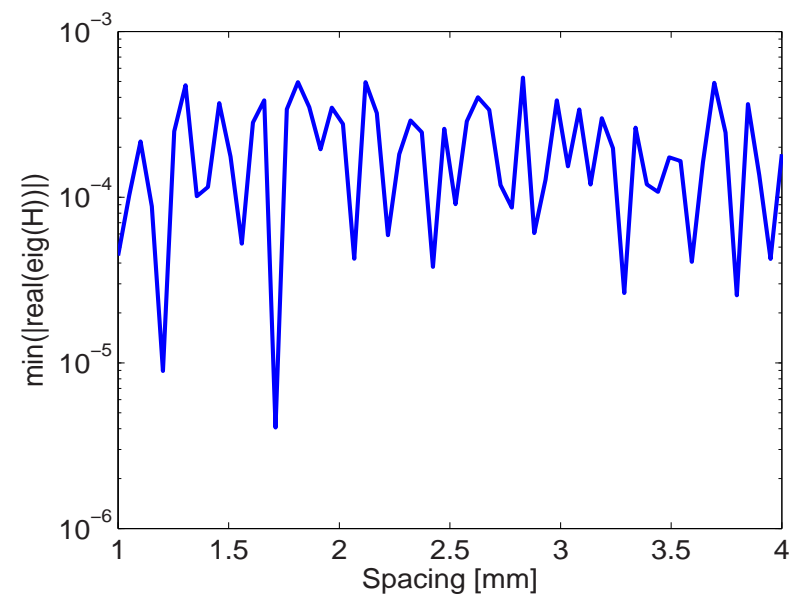

Fig. 7. Minimum absolute value of the real part of the Hamiltonian matrix eigenvalues.

of $L_{x}$ and 6 values of $L_{y}$ and with a CPU time equal to $43.7 \mathrm{~s}$. Then, the trivariate $\mathrm{ROM} \mathbf{Y}_{r}\left(s, L_{x}, L_{y}\right)$ is obtained with a reduced order $q=91$. Figs. 9-10 show the magnitude of the parameterized $\mathrm{ROM}$ of $\mathbf{Y}_{11}\left(s, L_{x}, L_{y}\right)$ for the vertical length values $L_{y}=\{0.46,0.93\} \mathrm{mm}$, while

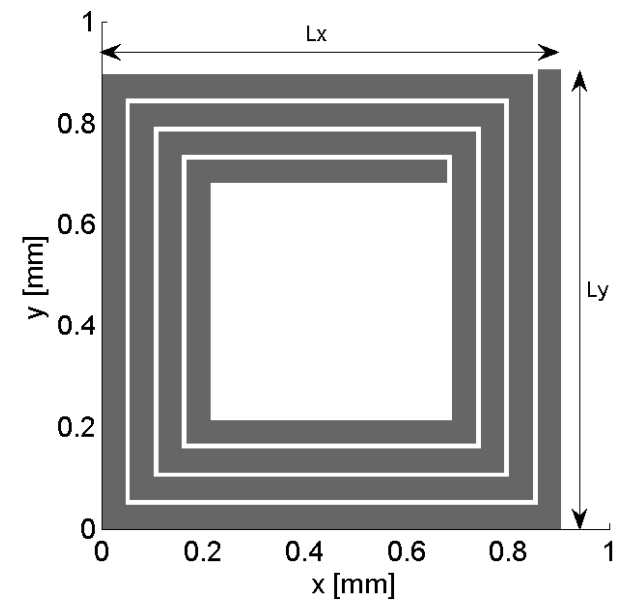

Fig. 8. Structure of the spiral inductor.

TABLE III

PARAMETERS OF THE SPIRAL INDUCTOR.

\begin{tabular}{|l|c|c|}
\hline Parameter & Min & Max \\
\hline Frequency (freq) & $10 \mathrm{kHz}$ & $30 \mathrm{GHz}$ \\
Horizontal length $\left(L_{x}\right)$ & $0.46 \mathrm{~mm}$ & $0.93 \mathrm{~mm}$ \\
Vertical length $\left(L_{y}\right)$ & $0.46 \mathrm{~mm}$ & $0.93 \mathrm{~mm}$ \\
\hline
\end{tabular}

Fig. 11 compares the magnitude of $\mathbf{Y}_{11}\left(s, L_{x}, L_{y}\right)$ and its parameterized ROM for the horizontal and vertical length values $L_{x}=0.63 \mathrm{~mm}, L y=\{0.50,0.63,0.76\} \mathrm{mm}$. These specific horizontal and vertical length values have not been used during the construction of the multivariate models $\overline{\mathbf{P}}(\boldsymbol{g}), \overline{\mathbf{L}}_{p}(\boldsymbol{g}), \overline{\mathbf{C}}_{d}(\boldsymbol{g}), \overline{\mathbf{R}}(\boldsymbol{g}), \overline{\mathbf{K}}_{r}(\boldsymbol{g})$, nevertheless an excellent agreement between model and data can be observed. The worst case RMS-error defined in (27) is equal to $5 \cdot 10^{-2}$ and it occurs for $\boldsymbol{g}_{\max }=\left\{L_{x}, L_{y}\right\}=\{0.86,0.76\} \mathrm{mm}$. Fig. 12 shows the minimum absolute value of the real part of the Hamiltonian matrix eigenvalues over a dense sweep of the design space. Since there are no purely imaginary eigenvalues, the parameterized ROM is passive over the design space of interest. As in the previous example, the parameterized ROM is able to accurately describe the parameterized behavior of the system, while preserving overall stability and passivity.

\section{Conclusions}

We have presented a new PMOR technique applicable to PEEC analysis which is based on a parameterization process of matrices generated by the PEEC method and the projection subspace generated by the Laguerre-SVD MOR method. Overall stability and passivity of parameterized ROMs are guaranteed by construction over the design space of interest. Numerical examples have validated the proposed PMOR approach on practical application cases, showing that it is able to build very accurate parameterized ROMs of highly dynamic EM systems, while guaranteeing stability and passivity over the entire design space of interest. 


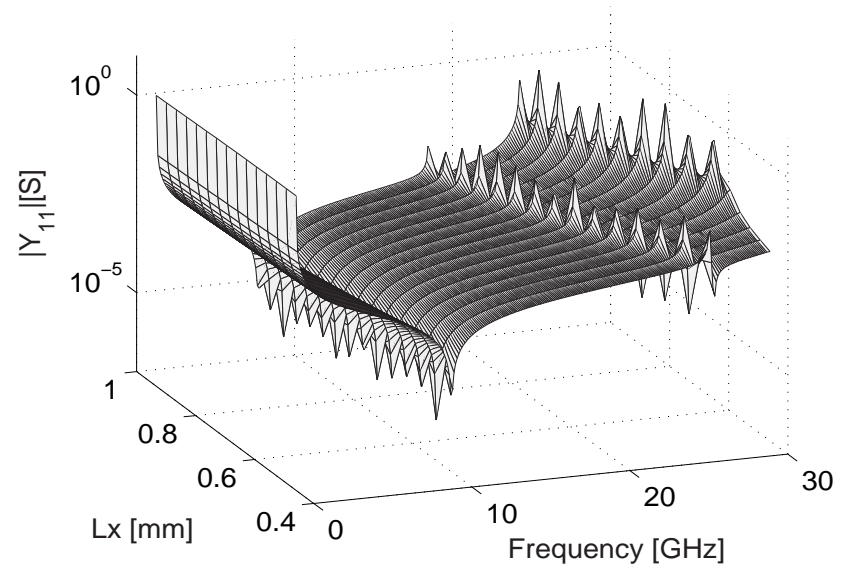

Fig. 9. Magnitude of the trivariate ROM of $\mathbf{Y}_{11}\left(s, L_{x}, L_{y}\right)\left(L_{y}=0.46\right.$ $\mathrm{mm})$.

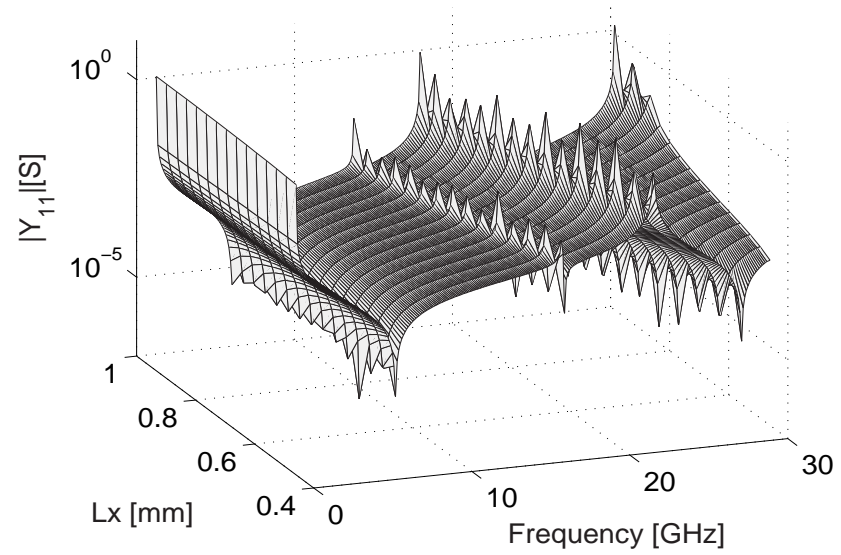

Fig. 10. Magnitude of the trivariate ROM of $\mathbf{Y}_{11}\left(s, L_{x}, L_{y}\right)\left(L_{y}=0.93\right.$ $\mathrm{mm})$.

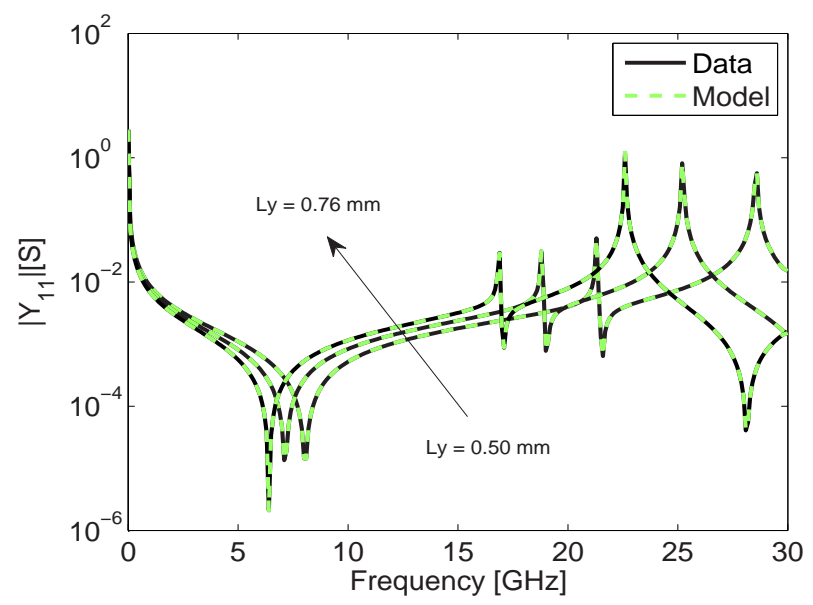

Fig. 11. Magnitude of the trivariate ROM of $\mathbf{Y}_{11}\left(s, L_{x}, L_{y}\right)\left(L_{x}=0.63\right.$ $\mathrm{mm}, L y=\{0.50,0.63,0.76\} \mathrm{mm})$.

\section{REFERENCES}

[1] R. F. Harrington, Field Computation by Moment Methods. New York: Macmillan, 1968.

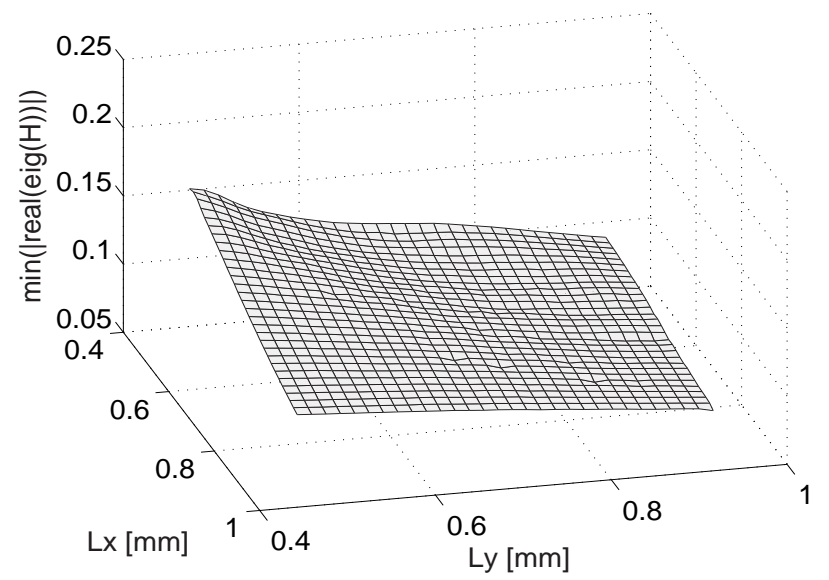

Fig. 12. Minimum absolute value of the real part of the Hamiltonian matrix eigenvalues.

[2] A. E. Ruehli, "Equivalent circuit models for three dimensional multiconductor systems," IEEE Trans. Microw. Theory Tech., vol. 22, no. 3, pp. 216-221, Mar. 1974.

[3] J. M. Jin, The Finite Element Method in Electromagnetics, 2nd ed. John Wiley and Sons, New York, 2002.

[4] K. Gallivan, E. Grimme, P. Van Dooren, "Asymptotic waveform evaluation via a Lanczos method," Applied Math., vol. 7, no. 5, pp. 75-80, Sep. 1994.

[5] P. Feldmann and R. W. Freund, "Efficient linear circuit analysis by Padé approximation via the Lanczos process," IEEE Trans. Comput.-Aided Design Integr. Circuits Syst., vol. 14, no. 5, pp. 639-649, May 1995.

[6] K. Gallivan, E. Grimme, P. Van Dooren, "A rational Lanczos algorithm for model reduction," Numerical Algorithms, vol. 12, pp. 33-63, 1996.

[7] A. Odabasioglu, M. Celik, and L. T. Pileggi, "PRIMA: passive reducedorder interconnect macromodeling algorithm," IEEE Trans. Comput.Aided Design Integr. Circuits Syst., vol. 17, no. 8, pp. 645-654, Aug. 1998.

[8] A. Dounavis, E. Gad, R. Achar, M. S. Nakhla, "Passive model reduction of multiport distributed interconnects," IEEE Trans. Microw. Theory Tech., vol. 48, no. 12, pp. 2325-2334, Dec. 2000.

[9] R. Achar, M. Nakhla, "Simulation of high-speed interconnects," Proc. IEEE, vol. 89, no. 5, pp. 693-728, May 2001.

[10] B. Denecker, F. Olyslager, L. Knockaert, and D. De Zutter, "Generation of FDTD subcell equations by means of reduced order modeling," IEEE Trans. Antennas Propag., vol. 51, no. 8, pp. 1806-1817, Aug. 2003.

[11] N. A. Marques, M. Kamon, L. M. Silveira, J. K. White, "Generating compact, guaranteed passive reduced-order models of 3-D RLC interconnects," IEEE Trans. Adv. Packag., vol. 27, no. 4, pp. 569-580, Nov. 2004.

[12] C. A. Balanis, Advanced Engineering Electromagnetics. John Wiley and Sons, New York, 1989.

[13] A. E. Ruehli and A. C. Cangellaris, "Progress in the methodologies for the electrical modeling of interconnects and electronic packages," Proc. IEEE, vol. 89, no. 5, pp. 740-771, May 2001.

[14] W. Pinello, A. C. Cangellaris, and A. Ruehli, "Hybrid electromagnetic modeling of noise interactions in packaged electronics based on the partial-element equivalent-circuit formulation," IEEE Trans. Microw. Theory Tech., vol. 45, no. 10, pp. 1889-1896, Oct. 1997.

[15] L. W. Nagel, "SPICE: A computer program to simulate semiconductor circuits," University of California, Berkeley, Electr. Res. Lab. Report ERL M520, May 1975.

[16] G. Antonini, "Fast multipole method for time domain PEEC analysis," IEEE Transactions on Mobile Computing, vol. 2, no. 4, pp. 275-287, October-December 2003.

[17] G. Antonini, A. E. Ruehli, "Fast multipole and multi-function PEEC methods," IEEE Transactions on Mobile Computing, vol. 2, no. 4, pp. 288-298, October-December 2003.

[18] R. D. Slone, W. T. Smith, Z. Bai, "Using partial element equivalent circuit full wave analysis and Padé via Lanczos to numerically simulate EMC problems," in Proc. IEEE Int. Symp. Electromagn. Compat., Austin, Tx, Aug. 1997, pp. 608-613. 
[19] L. Knockaert and D. De Zutter, "Laguerre-SVD reduced-order modeling," IEEE Trans. Microw. Theory Tech., vol. 48, no. 9, pp. 1469-1475, Sep. 2000

[20] Y. Liu, L. T. Pileggi, and A. J. Strojwas, "Model order-reduction of RC(L) interconnect including variational analysis," in Proc. IEEE/AMC Des. Autom. Conf., 1999, pp. 201-206.

[21] P. Heydari and M. Pedram, "Model reduction of variable-geometry interconnects using variational spectrally-weighted balanced truncation," in Proc. IEEE/ACM Int. Conf. Comput.-Aided Des., Nov. 4-8, 2001, pp. 586-591.

[22] L. Daniel, O. C. Siong, L. S. Chay, K. H. Lee, and J. White, "A multiparameter moment-matching model-reduction approach for generating geometrically parameterized interconnect performance models," IEEE Trans. Comput.-Aided Design Integr. Circuits Syst., vol. 23, no. 5, pp. 678-693, May 2004.

[23] X. Li, P. Li, and L. T. Pileggi, "Parameterized interconnect order reduction with explicit-and-implicit multi-parameter moment matching for inter/intra-die variations," in Proc. IEEE/ACM Int. Conf. Comput.Aided Des., Nov. 6-10, 2005, pp. 806-812.

[24] Y.-T. Li, Z. Bai, Y. Su, and X. Zeng, "Model order reduction of parameterized interconnect networks via a two-directional arnoldi process," IEEE Trans. Comput.-Aided Design Integr. Circuits Syst., vol. 27, no. 9 , pp. 1571-1582, Sep. 2008.

[25] G. Wollenberg, A. Görisch, "Analysis of 3-D interconnect structures with PEEC using SPICE,' IEEE Trans. Electromagn. Compat., vol. 41, no. 2, pp. 412-417, Nov. 1999.

[26] G. Antonini, A. Orlandi, "A wavelet based time domain solution for PEEC circuits," IEEE Trans. Circuits Syst., vol. 47, no. 11, pp. 1634 1639, Nov. 2000.

[27] A. E. Ruehli, G. Antonini, J. Esch, J. Ekman, A. Mayo and A. Orlandi, "Non-orthogonal PEEC formulation for time and frequency domain EM and circuit modeling," IEEE Trans. Electromagn. Compat., vol. 45, no. 2, pp. 167-176, May 2003.

[28] G. Antonini, A. Ruehli, and C. Yang, "PEEC modeling of dispersive and lossy dielectrics," IEEE Trans. Adv. Packag., vol. 31, no. 4, pp. 768 -782 , Nov. 2008.

[29] A. E. Ruehli, H. Heeb, "Circuit models for three-dimensional geometries including dielectrics," IEEE Trans. Microw. Theory Tech., vol. 40, no. 7, pp. 1507-1516, July 1992.

[30] A. E. Ruehli and H. Heeb, "Circuit models for three-dimensional geometries including dielectrics," IEEE Trans. Microw. Theory Tech., vol. 40, no. 7, pp. 1507-1516, Jul. 1992.

[31] C. Ho, A. Ruehli, P. Brennan, "The modified nodal approach to network analysis," IEEE Trans. Circuits Syst., vol. 22, no. 6, pp. 504-509, Jun. 1975.

[32] P. A. Brennan, A. E. Ruehli, "Capacitance models for integrated circuit metallization wires," IEEE Transactions on Solid State Circuits, vol. 10, no. 6, pp. 530-536, Dec. 1975

[33] R. W. Freund, "Krylov-subspace methods for reduced-order modeling in circuit simulation," J. Comput. Appl. Math., vol. 123, no. 1-2, pp. 395-421, 2000.

[34] R. Rohrer, H. Nosrati, "Passivity considerations in stability studies of numerical integration algorithms," IEEE Trans. Circuits Syst., no. 9, pp. 857-866, Sep. 1981.

[35] L. Weinberg, Network Analysis and Synthesis. New York: McGraw-Hill Book Company, 1962.

[36] E. A. Guillemin, Synthesis of Passive Networks. John Wiley and Sons, New York, 1957.

[37] J. De Geest, T. Dhaene, N. Faché and D. De Zutter, "Adaptive CADmodel building algorithm for general planar microwave structures," IEEE Trans. Microw. Theory Tech., vol. 47, no. 9, pp. 1801-1809, Sep. 1999.

[38] G. H. Golub and C. F. Van Loan, Matrix Computation. Baltimore, MD: John Hopkins, 1996.

[39] G. Allasia, "Simultaneous interpolation and approximation by a class of multivariate positive operators," Numerical Algorithms, vol. 34, no. 2, pp. 147-158, Dec. 2003.

[40] D. Shepard, "A two-dimensional interpolation function for irregularlyspaced data," in Proceedings of the 23rd ACM national conference. New York, NY, USA: ACM, 1968, pp. 517-524.

[41] A.Weiser and S. E. Zarantonello, "A note on piecewise linear and multilinear table interpolation in many dimensions," Mathematics of Computation, vol. 50, no. 181, pp. 189-196, Jan. 1988.

[42] C. de Boor, A Practical Guide to Splines. Springer-Verlag, 1992.

[43] M. Sakai and J. W. Schmidt, "Positive interpolation with rational splines," BIT, vol. 29, no. 1, pp. 140-147, 1989.
[44] M. Sarfraz, M. Z. Hussain, and S. Butt, "A rational spline for visualizing positive data," in Proc. IEEE Intern. Conf. on Information Visualization, Jul. 19-21, 2000, pp. 57-62.

[45] M. Z. Hussain and J. M. Ali, "Positivity-preserving piecewise rational cubic interpolation," Matematika, vol. 22, no. 2, pp. 147-153, 2006.

[46] J. Galliver, Geometric Methods and Applications: For Computer Science and Engineering. Springer, 2000.

[47] D. S. Bernstein, Matrix Mathematics: Theory, Facts, and Formulas: Second Edition. Princeton University Press, 2009.

[48] M. G. Safonov, R. Y. Chiang, and D. J. N. Limebeer, "Hankel model reduction without balancing - A descriptor approach," in Proc. 26th IEEE Conference on Decision and Control, vol. 26, Dec. 1987, pp. $112-117$.

[49] S. Boyd, L. El Ghaoui, E. Feron and V. Balakrishnan, Linear Matrix Inequalities in System and Control Theory. Philadelphia: SIAM, 1994.

[50] R. N. Shorten, P. Curran, K. Wulff, and E. Zeheb, "A note on spectral conditions for positive realness of transfer function matrices," IEEE Trans. Autom. Control, vol. 53, no. 5, pp. 1258-1261, Jun. 2008.

[51] "Matlab User's Guide," The Mathworks, Inc., Natick, 2009.

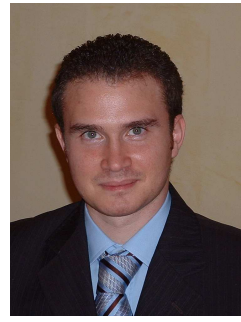

Francesco Ferranti (M'10) received the B.S. degree (summa cum laude) in electronic engineering from the Università degli Studi di Palermo, Palermo, Italy, in 2005 and the M.S. degree (summa cum laude and honors) in electronic engineering from the Università degli Studi dell'Aquila, L'Aquila, Italy, in 2007. He is currently working towards the Ph.D. degree in the Department of Information Technology (INTEC), Ghent University, Ghent, Belgium. His research interests include parametric macromodeling, parameterized model order reduction, EMC numerical modeling, system identification.

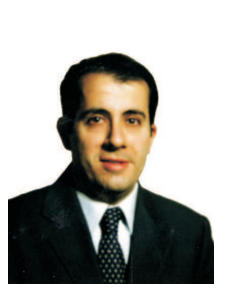

Giulio Antonini (M'94, SM'05) received his Laurea degree (summa cum laude) in Electrical Engineering in 1994 from the Università degli Studi dell'Aquila and the Ph.D. degree in Electrical Engineering in 1998 from University of Rome "La Sapienza". Since 1998 he has been with the UAq EMC Laboratory, Department of Electrical Engineering of the University of L'Aquila where he is currently Associate Professor. His research interests focus on EMC analysis, numerical modeling and in the field of signal integrity for high-speed digital systems. He has authored or co-authored more than 170 technical papers and 2 book chapters. Furthermore, he has given keynote lectures and chaired several special sessions at international conferences. He has been the recipient of the IEEE Transactions on Electromagnetic Compatibility Best Paper Award in 1997, the CST University Publication Award in 2004, the IBM Shared University Research Award in 2004, 2005 and 2006, the IET-SMT Best Paper Award in 2008. In 2006 he has received a Technical Achievement Award from the IEEE EMC Society "for innovative contributions to computational electromagnetic on the Partial Element Equivalent Circuit (PEEC) technique for EMC applications". He holds one European Patent. 


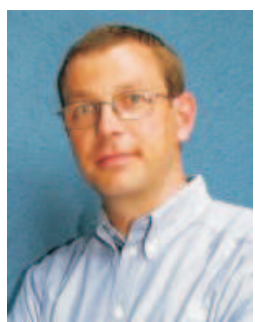

Tom Dhaene (SM'05) was born in Deinze, Belgium, on June 25, 1966. He received the Ph.D. degree in electrotechnical engineering from the University of Ghent, Ghent, Belgium, in 1993. From 1989 to 1993, he was Research Assistant at the University of Ghent, in the Department of Information Technology, where his research focused on different aspects of full-wave electro-magnetic circuit modeling, transient simulation, and time-domain characterization of high-frequency and high-speed interconnections. In 1993, he joined the EDA company Alphabit (now part of Agilent). He was one of the key developers of the planar EM simulator ADS Momentum. Since September 2000, he has been a Professor in the Department of Mathematics and Computer Science at the University of Antwerp, Antwerp, Belgium. Since October 2007, he is a Full Professor in the Department of Information Technology (INTEC) at Ghent University, Ghent, Belgium. As author or co-author, he has contributed to more than 150 peer-reviewed papers and abstracts in international conference proceedings, journals and books. He is the holder of 3 US patents.

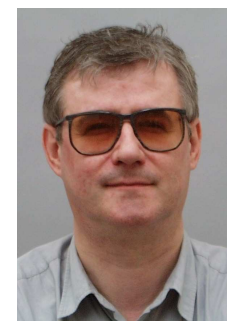

Luc Knockaert (SM'00) received the M. Sc. Degree in physical engineering, the M. Sc. Degree in telecommunications engineering and the $\mathrm{Ph}$. D. Degree in electrical engineering from Ghent University, Belgium, in 1974, 1977 and 1987, respectively. From 1979 to 1984 and from 1988 to 1995 he was working in North-South cooperation and development projects at the Universities of the Democratic Republic of the Congo and Burundi. $\mathrm{He}$ is presently affiliated with the Interdisciplinary Institute for BroadBand Technologies (www.ibbt.be) and a professor at the Dept. of Information Technology, Ghent University (www.intec.ugent.be). His current interests are the application of linear algebra and adaptive methods in signal estimation, model order reduction and computational electromagnetics. As author or co-author he has contributed to more than 100 international journal and conference publications. He is a member of MAA, SIAM and a senior member of IEEE.

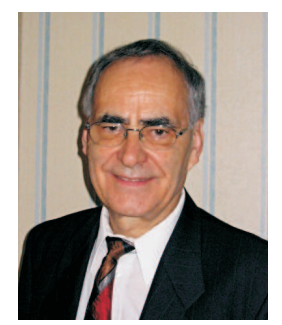

Albert E. Ruehli (LF'03) received the Ph.D. degree in electrical engineering from the University of Vermont, Burlington, in 1972, and an honorary doctorate from the Lulea University, Sweden, in 2007. He has been a member of various projects with IBM including mathematical analysis, semiconductor circuits and devices modeling, and as manager of a VLSI design and CAD group. Since 1972, he has been at IBM's T. J. Watson Research Center in Yorktown Heights, New York, where he was a Research Staff Member in the Electromagnetic Analysis Group. He is now an Emeritus of IBM Research and an adjunct professor in the EMC area at the Missouri University of Science and Technology. He is the editor of two books, Circuit Analysis, Simulation and Design (New York: North Holland 1986, 1987) and he is an author or coauthor of over 190 technical papers. Dr. Ruehli has served in numerous capacities for the IEEE. In 1984 and 1985, he was the Technical and General Chairman, respectively, of the ICCD International Conference. He has been a member of the IEEE ADCOM for the Circuit and System Society and an Associate Editor for the Transactions on Computer-Aided Design. He has given talks at universities including keynote addresses and tutorials at conferences, and has organized many sessions. He received IBM Research Division or IBM Outstanding Contribution Awards in 1975, 1978, 1982, 1995, and 2000. In 1982, he received the Guillemin-Cauer Prize Award for his work on waveform relaxation, and in 1999, he received a Golden Jubilee Medal, both from the IEEE CAS Society. In 2001, he received a Certificate of Achievement from the IEEE EMC Society for Inductance Concepts and the Partial Element Equivalent Circuit (PEEC) method. He received the 2005 Richard R Stoddart Award, and in 2007 he received the Honorary Life Member Award from the IEEE Electromagnetic Compatibility Society for outstanding technical performance. He is a member of SIAM. 